\title{
Impacto na Sobrevida Livre de Progressão pela Falta de Acesso a Inibidores de EGFR em Carcinoma de Pulmão de Células não Pequenas no Sistema de Saúde Público Brasileiro
}

\author{
Impact that Lack of Access to EGFR Inhibitors has on Progression-Free Survival in Non-Small Cell Lung Cancer treated via the \\ Public Health Care System in Brazil \\ Impacto en la Sobrevida Libre de Progresión por la Falta de Acceso a los Inhibidores de EGFR en el Carcinoma de \\ Pulmón de Células no Pequeñas en el Sistema de Salud Pública Brasileña
}

Gabriel Lenz ; Rodrigo Azevedo Pellegrini'; Lana Becker Micheletto'; Leonardo Stone Lago²

Resumo

Introduçáo: $\mathrm{O}$ advento de terapias-alvo antirreceptor do fator de crescimento epidérmico (anti-EGFR) impactou na sobrevida dos pacientes com câncer de pulmão de células não pequenas avançado e portadores de mutação no EGFR, que são tratados no Sistema Único de Saúde Brasileiro (SUS). Objetivo: Estimar o impacto da falta de acesso a terapias anti-EGFR na sobrevida livre de progressão (SLP) desses pacientes. Método: Por meio da base de dados do Instituto Nacional de Câncer José Alencar Gomes da Silva e de estudos que descrevem a prevalência de mutação em EGFR na população brasileira, foi estimado o número de pacientes com adenocarcinoma de pulmão avançado, portadores de mutação EGFR, candidatos à terapia-alvo no ano de 2017. Para a estimativa de efetividade, quatro diferentes esquemas de tratamentos foram considerados: quimioterapia, erlotinib, afatinib e gefitinib. O número de pacientes livres de progressão de doença, após dois anos, foi estimado com base nos resultados para SLP em ensaios clínicos randomizados. Resultados: Foram estimados 1.735 pacientes com adenocarcinoma de pulmão metastático portadores de mutaçóes ativadoras de EGFR no Brasil para o ano de 2017. Projetou-se que, caso fossem tratados com quimioterapia, apenas 71 estariam livres de progressão após 24 meses do início do tratamento. Em contrapartida, com o uso de inibidores de tirosina-quinase anti-EGFR, a expectativa seria de 312 pacientes livres de doença para erlotinib, 377 para gefitinib e 388 para afatinib. Conclusáo: Apesar de recomendadas internacionalmente, as terapias anti-EGFR náo são disponibilizadas no SUS, sendo oferecida aos pacientes apenas a quimioterapia. Isso problematiza a situação de falta de acesso no âmbito do SUS e embasa, localmente, a discussão acerca da incorporação dessas terapias no âmbito público.

Palavras-chave: Neoplasias Pulmonares; Sistema Único de Saúde; Genes erbB-1.

\begin{abstract}
Introduction: The advent of targeted anti-epidermal growth factor receptor (anti-EGFR) therapies have improved survival in patients with metastatic non-small cell lung cancer that carry the EGFR mutation, including those treated via the Brazilian Sistema Único de Saúde (SUS, Unified Health Care System). Objective: To estimate the impact that lack of access to anti-EGFR therapies has on progression-free survival (PFS) among such patients. Method: On the basis of epidemiologic data obtained from the José Alencar Gomes da Silva National Cancer Institute and from studies reporting the prevalence of the EGFR mutation in the Brazilian population, we estimated the number of patients with advanced lung adenocarcinoma and the EGFR mutation who were candidates for targeted therapy in 2017. To estimate effectiveness, we evaluated four different treatments: chemotherapy, erlotinib, afatinib, and gefitinib. The number of patients with PFS after 2 years of follow-up was estimated on the basis of the results of randomized clinical trials. Results: We evaluated 1,735 patients with EGFR mutation-positive metastatic lung adenocarcinoma in Brazil in 2017. We estimated that, if treated with chemotherapy, only 71 of those patients would be free of progression after 24 months. In contrast, if all of the patients were treated with anti-EGFR tyrosine kinase inhibitors, the expectation was that PFS would be achieved in 312 patients for erlotinib, 377 for gefitinib, and 388 for afatinib. Conclusion: Although recommended by international guidelines, anti-EGFR therapies are not available via the SUS, which offers only chemotherapy. This complicates the problem of lack of access in the SUS and promotes local discussion in the public sphere about the incorporation of these therapies.

Key words: Lung Neoplasms; Unified Health Care System; Genes, erbB-1.
\end{abstract}

Resumen

Introducción: El advenimiento de terapias objetivo anti receptor del factor de crecimiento epidérmico (EGFR) impactó en la supervivencia de los pacientes con cáncer de pulmón de células no pequeñas avanzado y portadores de mutación en el EGFR, que son tratados en el sistema único de salud brasileño (SUS). Objetivo: Estimar el impacto de la falta de acceso a terapias anti-EGFR en la sobrevida libre de progresión (SLP) de esos pacientes. Método: A través de la base de datos del Instituto Nacional de Cáncer José Alencar Gomes da Silva y de estudios que describen la prevalencia de mutación de EGFR en la población brasileña, se estima el número de pacientes con adenocarcinoma de pulmón avanzado portadores de mutación EGFR candidatos a la terapia objetivo en el año de Para la estimación de efectividad, cuatro diferentes esquemas de tratamientos fueron considerados: quimioterapia, erlotinib, afatinib y gefitinib. El número de pacientes libres de progresión de la enfermedad después de dos ańos se calculó sobre la base de los resultados para SLP en los ensayos clínicos aleatorizados. Resultados: Se estimó 1.735 pacientes con adenocarcinoma de pulmón metastático, portadores de mutaciones activadoras de EGFR en Brasil para el año 2017. Se proyectó que si se tratar con quimioterapia sólo 81 estarían libres de progresión después de 24 meses. En contrapartida, con el uso de inhibidores de tirosina quinasa anti-EGFR, la expectativa sería de 312 pacientes libres de enfermedad para erlotinib, 377 para gefitinib y 388 para afatinib. Conclusión: A pesar de ser recomendadas internacionalmente, las terapias anti-EGFR no están disponibles en el SUS, siendo ofrecido a los pacientes sólo quimioterapia. Esto problematiza claramente la situación de falta de acceso en el ámbito del SUS y basan, localmente, la discusión sobre la incorporación de estas terapias en el ámbito público.

Palabras clave: Neoplasias Pulmonares; Sistema Único de Salud; Genes erbB-1.

${ }^{1}$ Estudante de Medicina. Escola de Medicina da Pontifícia Universidade Católica do Rio Grande do Sul (PUC-RS). Porto Alegre (RS), Brasil.

${ }_{2}$ Médico-Oncologista no Hospital Santa Rita de Porto Alegre. Porto Alegre (RS), Brasil.

Endereço para correspondência: Gabriel Lenz. Rua dos Burgueses, 295 - Apto. 1702. Porto Alegre (RS), Brasil. CEP 91530-020 . E-mail: gabriel.lenz@acad.pucrs.br. 


\section{INTRODUÇÃO}

O câncer de pulmão é a neoplasia maligna líder em mortalidade mundialmente ${ }^{1}$. Esse tumor, no Brasil, figura como um importante problema de saúde pública, com estimativas anuais de 28.220 novos casos e 24.490 mortes $^{2}$.

$\mathrm{O}$ carcinoma de pulmão de células não pequenas (CPCNP) representa $85 \%$ das neoplasias primárias de pulmão, sendo a maior parte dos pacientes diagnosticados em estágios avançados $(44 \%)^{1,3}$. Para esses pacientes, o tratamento sistêmico paliativo destaca-se como a principal opção terapêutica. Com o avanço das técnicas de diagnóstico molecular, terapias-alvo mais efetivas e menos tóxicas do que a quimioterapia estão, hoje, disponíveis para pacientes com mutaçóes ativadoras do receptor do fator de crescimento epidérmico (EGFR) e translocação de quinase do linfoma anaplásico (ALK), por exemplo ${ }^{4}$. Em estudo publicado por Midha et al., a prevalência de mutações ativadoras do EGFR na população brasileira foi estimada em $28 \%$ nos pacientes com adenocarcinoma de pulmão avançado 5 .

Desde a comprovação da maior eficácia e menor toxicidade dos inibidores de tirosina-quinase (TKI) anti-EGFR em comparação com a quimioterapia citotóxica no tratamento de primeira linha paliativa nessa subpopulação, a agência regulatória Norte-Americana Food and Drug Administration (FDA) aprovou diferentes drogas para esse propósito: o erlotinib, em maio de 2013; o afatinib, em julho de 2013; e o gefitinib, em junho de $2015^{6,7}$. Entretanto, destaca-se que os estudos clínicos que avaliaram essas drogas demonstraram benefícios significativos na sobrevida livre de progressão (SLP), porém sem aumento na sobrevida global (SG) quando comparados à quimioterapia padráo ${ }^{8}$.

O acesso aos TKI, contudo, por meio do Sistema Único de Saúde do Brasil (SUS), é restrito. Apesar de aprovado pela Comissão Nacional de Incorporação de Tecnologias no SUS (Conitec) para pacientes com CPCNP em estágio IV com mutação de EGFR, essas drogas não estão efetivamente disponíveis aos seus usuários, em razão da ausência de orçamento e repasse específico que cubram os custos, com exceção do estado de São Paulo. Dessa forma, o acesso dos pacientes a essas medicações se dá por intermédio de ações judiciais. O presente estudo tem como objetivo estimar o impacto da falta de acesso a terapias anti-EGFR em termos de SLP em pacientes com CPCNP metastáticos com mutaçóes ativadoras de EGFR tratados no âmbito do SUS.

\section{MÉTODO}

O número de pacientes candidatos a receberem terapia com TKI em 2017 foi estimado a partir dos dados de prevalência e incidência de neoplasia pulmonar divulgados pelo Instituto Nacional de Câncer José Alencar Gomes da Silva (INCA), no relatório de estimativa de incidência de câncer para o biênio 2016-2017². A parcela da população com acesso a planos de saúde suplementar, seja privada ou para servidores públicos, com base em dados da pesquisa de saúde de 2013 do Instituto Brasileiro de Geografia e Estatística (IBGE), foi excluída da análise, tendo em vista não se tratar do público-alvo dessa análise? ${ }^{9}$ Como os dados de prevalência de mutaçóes ativadoras do EGFR, necessária para o uso de TKI, referem-se à histologia adenocarcinoma, apenas esse subtipo foi considerado 5 . $\mathrm{O}$ número estimado de pacientes com doença metastática foi observado a partir da estimativa da soma do número de pacientes diagnosticados em estádio IV (EC IV) ${ }^{3}$ no ano de 2017, com a estimativa do número de casos diagnosticados nos cinco anos anteriores com recidiva em 2017. Para esse fim, foi aplicada uma taxa de recidiva em cinco anos de pacientes diagnosticados em EC I, II e III com base nos seus prognósticos ${ }^{10}$.

A incidência de pacientes brasileiros portadores de adenocarcinoma com mutação em EGFR foi obtida com base no estudo publicado por Midha et al. ${ }^{5} \mathrm{~A}$ Figura 1 sumariza a metodologia utilizada para estimar o contingente de pacientes com câncer de pulmão avançado candidatos à terapia anti-EGFR.

A estimativa do número de pacientes livres de progressão, após dois anos, foi obtida aplicando-se as estimativas das taxas de SLP de doença após 24 meses de acompanhamento, calculadas com base nos resultados reportados pelos estudos Eurtac (erlotinib), LUX-Lung 3 (afatinib) e LUX-Lung 7 (gefitinib) ${ }^{11-13}$. A taxa de SLP para quimioterapia foi obtida levando-se em consideração

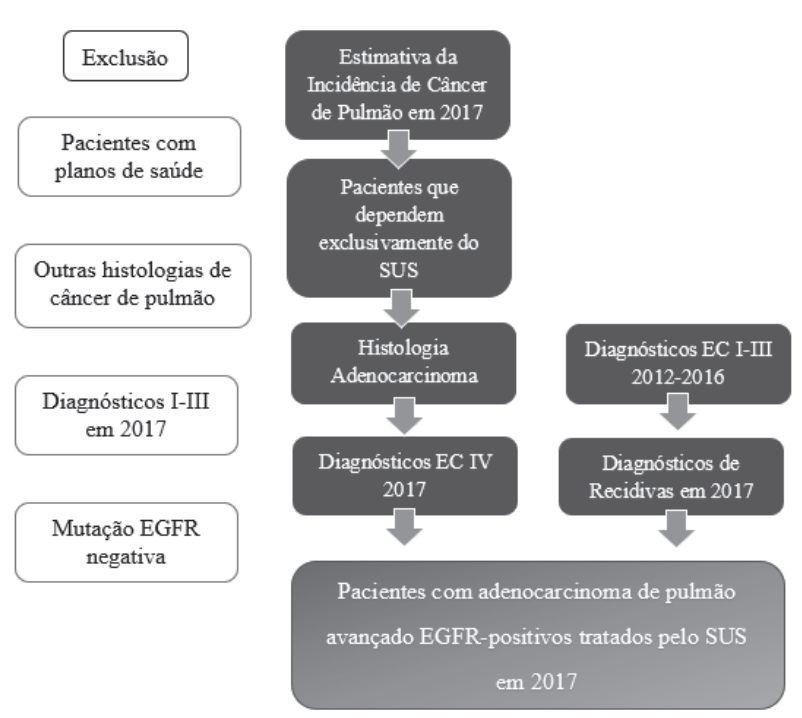

Figura 1. Pacientes com adenocarcinoma de pulmão avançado EGFR-positivos tratados pelo SUS em 2017 
o braço controle (poliquimioterapia com platina) do estudo Eurtac ${ }^{11}$.

O delineamento do estudo é de um artigo original misto, que foi elaborado por meio de estimativas e cálculos estatísticos, sempre baseados em dados previamente publicados. E, a partir disso, embasados na resoluçáo 466 da Plataforma Brasil, não houve necessidade de aprovação do Comitê de Ética em Pesquisa (CEP).

\section{RESULTADOS}

O INCA estima 28.220 novos casos de câncer de pulmão anualmente no Brasil ${ }^{2}$. Desses, $76,3 \%$ são tratados no SUS, totalizando 21.532 casos ${ }^{9}$. Verificou-se, então, conforme a Sociedade Americana de Câncer, que $40 \%$ dos pacientes seriam portadores de adenocarcinomas ( $40 \%$ de $21.532=8.613$ pacientes), dos quais $44 \%$ se apresentam em EC IV (44\% de $8.613=3.790$ pacientes) $)^{3,14}$. Foram somados a esse resultado os pacientes diagnosticados em estágios I a III, nos cinco anos anteriores, e que tendem a desenvolver recidiva em 2017. Para o EC III, tem-se uma taxa de recorrência em cinco anos de 53,79\%; para o EC II, de $46,49 \%$; e para o EC I, de $26,06 \%{ }^{10}$. Com base nessas taxas, estimaram-se 2.407 pacientes com recidivas diagnosticadas em 2017 que, somados àqueles diagnosticados em EC IV, totalizam 6.197 casos de adenocarcinoma de pulmão avançados atendidos pelo SUS no ano. Destes, assumiu-se que $28 \%$ possuiriam mutação ativadora em EGFR, resultando em 1.735 pacientes candidatos à terapia anti-EGFR ${ }^{5}$. A Tabela 1 sumariza didaticamente esses resultados.

Assumindo-se que todos esses pacientes recebam poliquimioterapia com platina (tratamento aprovado pelo SUS) e aplicando-se uma taxa de $12,48 \%$ para a quantidade mensal de pacientes que têm progressão de doença (a mesma observada no estudo Eurtac), apenas 71 indivíduos estariam livres de progressão após dois anos. Para essa última estimativa, foi considerada como constante a fração de pacientes que têm progressão de doença ao longo do tratamento.

Em contrapartida, estima-se um contingente maior de indivíduos livres de progressão da doença, nesse mesmo período de seguimento, com base na mesma metodologia, caso fossem disponibilizados os tratamentos de escolha para essa população: 377 com o uso do gefitinib $(6,16 \%$ de progressão mensal); 388 com afatinib (6,05\%); e 312 com o erlotinib (6,9\%) (Figura 2).

\section{DISCUSSÃO}

A terapia-alvo é uma descoberta do século passado no combate ao câncer. A ideia de selecionar uma molécula específica da célula cancerígena como foco terapêutico
Tabela 1. Estimativa do total de pacientes com câncer de pulmão não pequenas células EGFR mutados aptos a receberem terapia-alvo de inibidores de tirosina-quinase no Brasil

\begin{tabular}{|c|c|}
\hline Variável & n (\%) \\
\hline $\begin{array}{l}\text { Dependência exclusiva do SUS } \\
\text { entre todos os casos reportados } \\
\text { de câncer de pulmão em } 2017 \\
(n=28.220)\end{array}$ & $21.532(76,30)$ \\
\hline $\begin{array}{l}\text { Casos de adenocarcinoma entre } \\
\text { pacientes dependentes do SUS } \\
(n=21.532)\end{array}$ & $8.613(40,00)$ \\
\hline $\begin{array}{l}\text { Estádio clínico no momento do } \\
\text { diagnóstico entre pacientes com } \\
\text { adenocarcinoma dependentes de } \\
\text { SUS }(n=8.613)\end{array}$ & \\
\hline $\begin{array}{l}\text { (a) Estágio IV (metástase ao } \\
\text { diagnóstico) }\end{array}$ & $3.790(44,0)$ \\
\hline Estágio III & $3703(43,0)$ \\
\hline Estágio II & $603(7,0)$ \\
\hline Estágio I & $517(6,0)$ \\
\hline $\begin{array}{l}\text { Recorrência em } 5 \text { anos por } \\
\text { estágio }\end{array}$ & \\
\hline (b) Estágio III (3.703) & $1.992(53,79)$ \\
\hline (c) Estágio II (603) & $280(46,49)$ \\
\hline (d) Estágio I (517) & $135(26,06)$ \\
\hline $\begin{array}{l}\text { Candidatos à terapia anti-EGFR } \\
\text { direcionada em pacientes com } \\
\text { adenocarcinoma avançado no } \\
\text { Brasil (a }+b+c+d=6.197 \text { ) }\end{array}$ & $1.735(28,00 *)$ \\
\hline
\end{tabular}

* Baseado em uma amostra de 239 pacientes com câncer de pulmáo de não pequenas células, avaliados por Midha et al. ${ }^{5}$

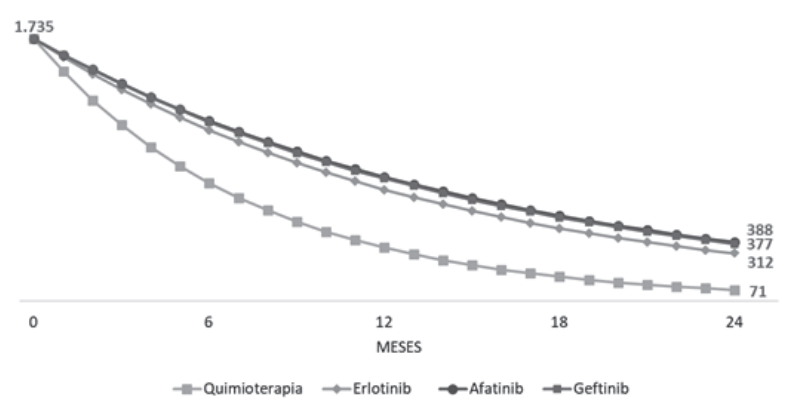

Figura 2. Curvas de estimativa para sobrevida livre de progressão em dois anos nos pacientes selecionados por grupo de tratamento: (i) quimioterapia, (ii) erlotinib, (iii) afatinib e (iv) gefitinib

data de 1997, com a aprovação, pelo FDA, do anticorpo monoclonal rituximab contra linfoma não Hodgkin, e tem como seu principal exponente o trastuzumab, outro anticorpo monoclonal, aprovado em 1999, para o tratamento do câncer de mama ${ }^{7}$. 
A molécula-alvo, nas neoplasias pulmonares, com maior relevância é o EGFR. Nesse molde, o estudo Eurtac, para câncer de pulmáo, obteve sucesso em TKI para pacientes com mutação nessa proteína ${ }^{11}$. Há menos de cinco anos, o FDA aprovou três drogas para o tratamento de primeira linha em pacientes que tenham câncer de pulmão de células não escamosas metastático e positivos para a mutação EGFR: erlotinib, afatinib e gefitinib ${ }^{7}$. Essas drogas, comparadas à quimioterapia, apresentaram aumento da SLP e diminuição da toxicidade ${ }^{6}$.

Com o advento de descobertas como essas, foi observado grande progresso no tratamento de câncer de pulmáo, ao longo das últimas décadas, com importante aumento na expectativa de vida desses pacientes ${ }^{15}$. Os dados do programa americano de Vigilância, Epidemiologia e Resultados (Seer) demonstram que as taxas de sobrevida desses pacientes em 12,24 e 60 meses aumentaram de 29,3\%, 16,4\% e 9\%, respectivamente, em 1973 , para $46,5 \%, 30 \%$ e $17 \%$, em $2013^{15}$. Por outro lado, o câncer de pulmão permanece como a neoplasia com maior mortalidade entre todos os sítios primários mundialmente ${ }^{16}$.

Os ensaios clínicos randomizados (ECR) usando TKI náo demonstraram ganho em SG nesse grupo de pacientes. Contudo, vale ressaltar que, apesar de não aumentar a SG, sabe-se atualmente que a SLP é aceita para avaliação e consequente aprovação de novas modalidades terapêuticas. Conforme literatura, ela é utilizada como desfecho primário em $51 \%$ dos estudos ${ }^{17}$. Além disso, há uma correlação estatisticamente significativa entre a SG e a SLP, sendo esta aceita como alternativa para a primeira ${ }^{18}$. Foi com base nesse argumento que diversos estudos utilizaram a SLP como desfecho primário em seus resultados, como bevacizumab (câncer de células renais) e crizotinib (câncer de pulmão) ${ }^{19,20}$.

Entretanto, na realidade brasileira, apenas uma minoria da população possui acesso a tratamentos mais complexos, principalmente na área oncológica. O sistema de saúde público do país possui duas formas de financiamento, uma pública, o SUS; e outra privada, que compreende o sistema suplementar de saúde, sendo que cada uma destas possui um peso de aproximadamente $50 \%$ no total de gastos em saúde ${ }^{21}$. Por outro lado, a porção da população que depende do financiamento público é de $74,3 \%$. A consequência desse modelo de financiamento, em que o mercado privado possui um gasto per capita três vezes o gasto do mercado público, gera uma inequidade na saúde brasileira.

Para o grupo de pacientes cobertos pelo sistema suplementar, as drogas, assim que regularizadas e aprovadas pela Agência Nacional de Vigilância Sanitária (Anvisa), logo são concedidas. Em relação à população dependente exclusivamente do sistema público, a realidade é outra. O Ministério da Saúde financia drogas oncológicas em dois formatos: compra centralizada e Autorização de Procedimentos Ambulatoriais de Alta Complexidade/ Custos (Apac). No primeiro caso, os medicamentos são fornecidos gratuitamente pelo próprio sistema, exemplo de trastuzumab e rituximab ${ }^{22}$. Em contrapartida, a Apac abrange a grande maioria dos fármacos. Nessa forma de repasses, há um valor fixo, previamente estabelecido pelo Ministério da Saúde, que financiará o tratamento realizado pelo hospital ${ }^{23,24}$.

É necessário que, em ambas as formas de financiamento do Ministério da Saúde para medicamentos oncológicos, haja a aprovaçáo por parte da Anvisa, a qual avalia eficácia e segurança da droga. Além disso, faz-se essencial também a permissão da Conitec, que realiza uma análise de custo-benefício para decidir se o tratamento deve ou não ser fornecido pelo Estado ${ }^{25}$.

De fato, assim como a Anvisa já havia feito, a Conitec aprovou a incorporaçáo do erlotinib e do gefitinib em novembro de 2013 ao SUS ${ }^{25}$. Entretanto, esses tratamentos dependem do modelo Apac de financiamento. $O$ ponto de interesse desse trabalho reside no fato de que, nessa forma de financiamento, há determinados casos em que o valor repassado ao hospital pelo tratamento oferecido não necessariamente cobre integralmente o gasto ao qual a instituição de saúde está submetida. Assim, configura-se um claro desincentivo à busca de tratamentos não devidamente financiados, sendo as drogas base deste estudo - gefitinib e erlotinib - um exemplo de tais práticas. Tal fato praticamente impossibilita que os hospitais públicos ofereçam esse tratamento aos pacientes que não tenham plano de saúde.

Dessa maneira, é vista certa divergência entre o que preconizam os protocolos internacionais no tratamento de pacientes com CPCNP metastáticos com mutação de EGFR e o que é oferecido aos cidadãos brasileiros dependentes do sistema público de saúde. Nessa perspectiva, o tratamento com quimioterapia padráo gera consequências em comparação aos resultados obtidos pós tratamento com TKI, sendo esse o principal fator que o estudo visou a elucidar.

Com esse intuito, este estudo evidenciou que, em relação aos diagnósticos no ano de 2017, até 388 pacientes estariam livres de progressão da doença, ao final de dois anos, utilizando as terapias-alvo para mutaçóes de EGFR, comparados a, apenas, 71 desses pacientes, quando submetidos ao tratamento fornecido pelo SUS.

Sabemos, hoje, que, além de médicos e pacientes, a indústria farmacêutica e instâncias governamentais muito influenciam para que uma droga seja incorporada universalmente. Nessa perspectiva, um estudo que tenta mostrar as consequências de privar uma população de determinada droga pode muito influenciar nessas escolhas. 
Esta análise apresenta limitações significativas. Os dados epidemiológicos do Brasil, por exemplo, são, muitas vezes, escassos, com isso foram usadas como base as estimativas do INCA. Ao mesmo tempo, por meio da literatura mundial, sabe-se que os $28 \%$ usados para mutação em EGFR são superestimados; porém, para fins de cálculos, esse foi o valor escolhido para nossa estimativa. Tratam-se também de dados de pacientes diagnosticados com doença avançada apenas. O prognóstico de recidivas teve como base um estudo americano, em que os tratamentos disponíveis para aquela população não são, obrigatoriamente, os mesmos para nossa população analisada. Além disso, estamos excedendo as diferenças de SLP diretamente de ECR, e é possível que essas diferenças não sejam tão pronunciadas na prática clínica. Essa suposição pode introduzir um viés favorecendo a diferença entre os tratamentos na vida real.

\section{CONCLUSÃO}

Em linhas gerais, seis anos após a publicação do estudo Eurtac, o SUS ainda náo disponibilizou definitivamente esses tratamentos na tabela de drogas fornecidas para a população. A introdução de tais fármacos poderia influenciar na SLP dessas pessoas. Com esse intuito, este estudo demonstrou que, em dois anos, poderia ser evitada a progressão da doença de 317 pacientes.

\section{CONTRIBUIÇÕES}

Gabriel Lenz e Leonardo Stone Lago trabalharam na concepção e desenho do trabalho; análise, interpretação dos dados da pesquisa; na redação, revisão crítica com contribuição intelectual e na aprovação final da versão para publicação. Rodrigo Azevedo Pellegrini trabalhou na concepção e desenho do trabalho; análise e interpretação dos dados da pesquisa. Lana Becker Micheletto trabalhou na redação e na revisão crítica com contribuição intelectual.

\section{DECLARAÇÃO DE CONFLITO DE INTERESSES}

Nada a Declarar.

\section{REFERÊNCIAS}

1. Yang X, Yang K, Kuang K. The efficacy and safety of EGFR inhibitor monotherapy in non-small cell lung cancer: a systematic review. Curr Oncol Rep. 2014;16(6):390.

2. Instituto Nacional de Câncer José Alencar Gomes da Silva. Estimativa 2016. Rio de Janeiro: Inca; 2015.

3. Instituto Nacional de Câncer José Alencar Gomes da Silva. IntegradorRHC: Ferramenta para a Vigilância Hospitalar de Câncer no Brasil. Rio de Janeiro: Inca; 2011.
4. Gridelli C, Peters S, Sgambato A, Casaluce F, Adjei AA, Ciardiello F. ALK inhibitors in the treatment of advanced NSCLC. Cancer Treat Rev. 2014;40(2):300-6.

5. Midha A, Dearden S, McCormack R. EGFR mutation incidence in non-small-cell lung cancer of adenocarcinoma histology: a systematic review and global map by ethnicity (mutMapII). Am J Cancer Res. 2015;5(9):2892-911.

6. Mok TS, Carbone DP, Hirsh FR (Editores). The IASLC Atlas of EGFR Testing in Lung Cancer. Aurora, CL: IASLC; 2017.

7. Department of Health and Human Services (EUA). Drugs@FDA: FDA Approved Drug Products; [Internet] [acesso em 2018 junho 7]. Disponível em: https://www. accessdata.fda.gov/scripts/cder/daf/.

8. Kobayashi S, Boggon TJ, Dayaram T, Jänne PA, Kocher O, Meyerson M, Johnson BE, Eck MJ, Tenen DG, Halmos B. EGFR mutation and resistance of non-small-cell lung cancer to gefitinib. N Engl J Med. 2005;352(8)786-92.

9. Instituto Brasileiro de Geografia e Estatística. Sistema IBGE de Recuperação Automática - SIDRA [Internet]. [acesso em 2018 abril 27]. Disponível em: https://sidra. ibge.gov.br/pesquisa/pns

10. Wong ML, McMurry TL, Stukenborg GJ, Francescatti $\mathrm{AB}$, Amato-Martz $\mathrm{C}$ et al. Lung Cancer Impact of age and comorbidity on treatment of non-small cell lung cancer recurrence following complete resection: a nationally representative cohort study. Lung Cancer. 2016;102:108-17.

11. Rosell R, Carcenery E, Gervais R, Vergnenegre A, Massuti B, Felip E et al. Erlotinib versus standard chemotherapy as fi rst-line treatment for European patients with advanced EGFR mutation-positive non-small-cell lung cancer (EURTAC): a multicentre, open-label, randomised phase 3 trial. Lancet Oncol. 2012;13(3):239-46.

12. Sequist LV, Yang JC, Yamamoto N, O'Bryrne K, Hirsh V, Mok T et al. Phase III study of afatinib or cisplatin plus pemetrexed in patients with metastatic lung adenocarcinoma with EGFR mutations. J Clin Oncol. 2013;31(27):3327-34

13. Park K, Tan EH, O’Byrne K, Zhang L, Boyer M, Mok $T$, Hirsh V, et al. Afatinib versus gefi tinib as fi rst-line treatment of patients with EGFR mutation-positive non-small-cell lung cancer (LUX-Lung 7): a phase 2B, open-label, randomised controlled trial. Lancet Oncol. 2016;17(5):577-89.

14. American Cancer Society. What Is Non-Small Cell Lung Cancer? [Internet]; 2016 [acesso em: 2017 set 5]. Disponível em: https://www.cancer.org/cancer/ non-small-cell-lung-cancer/about/what-is-non-smallcell-lung-cancer.html

15. National Cancer Institute. Cancer Statistics Review (CRS) 1975-2014 [Internet]; 2018 [acesso em: 2018 mar 15]. Disponível em: https://seer.cancer.gov/ csr/1975_2014 
16. Kang P, Liao M, Wester MR, Leeder JS. Pearce RE, Correia MA. CYP3A4-Mediated carbamazepine (CBZ) metabolism: formation of a covalent CBZ-CYP3A4 adduct and alteration of the enzyme kinetic profile. Drug Metab Dispos. 2008;36(3):490-9.

17. Diniz RW, Guerra MR, Teixeira MTB. Sobrevida Livre de Doença e Fatores Associados em Pacientes com Câncer de Mama não Metástico [dissertação]. Juiz de Fora: Universidade Federal de Juiz de Fora; 2014.

18. Machado KK, Katz A, Buyse M, Saad ED. Sobrevida global e outros desfechos clínicos em câncer de mama: situação atual e controvérsias. Rev Assoc Med Bras. 2010;56(5):514-6.

19. Escudier B, Pluzanska A, Koralewski P, Ravaud A, Bracarda S, Szczylik C, et al. Bevacizumab plus interferon alfa-2a for treatment of metastatic renal cell carcinoma: a randomised, double-blind phase III trial. Lancet. 2016;370(9605): 2103-11.

20. Shaw AT, Kim DW, Nakagawa K, Seto T, Crinó L, Ahn MJ, De Pas T, et al. Crizotinib versus Chemotherapy in Advanced ALK-Positive Lung Cancer. N Engl J Med. 2013;368(25):2385-94.

21. World Bank Group. International Development, Poverty \& Sustainability [Internet] [acesso em 2017 nov 2]. Disponível em: http://www.worldbank.org/

22. Ministério da Saúde (BR). Portaria $n^{\circ} 73$, de 30 de janeiro de 2013. Inclui procedimentos na Tabela de Procedimentos, Medicamentos, Órteses/Próteses e Materiais Especiais do SUS e estabelece protocolo de uso do trastuzumabe na quimioterapia do câncer de mama HER-2 positivo inicial e localmente avançado. Diário Oficial [da] União, Brasília, DF; 2013.

23. Comissão Nacional de Incorporação de Tecnologias no SUS (BR). Erlotinibe para câncer de pulmão de células não pequenas. Brasília, DF: Conitec; 2013.

24. Comissão Nacional de Incorporação de Tecnologias no SUS (BR). Gefitinibe para câncer de pulmão de células não pequenas em primeira linha. Brasília, DF: Conitec; 2013.

25. Comissão Nacional de Incorporação de Tecnologias no SUS (BR). Página inicial [Internet] [acesso em: 2017 jul 6]. Disponível em: http://conitec.gov.br/ 